\title{
BMJ Open Inflammatory blood parameters as prognostic factors for surgical site infection after primary hip or knee arthroplasty: a systematic review protocol
}

\author{
Petr Domecky (D) , Anna Rejman Patkova (D) , Katerina Mala-Ladova (D) , \\ Josef Maly
}

To cite: Domecky P, Rejman Patkova A, Mala-Ladova K, et al. Inflammatory blood parameters as prognostic factors for surgical site infection after primary hip or knee arthroplasty: a systematic review protocol. BMJ Open 2021;11:e046027. doi:10.1136/ bmjopen-2020-046027

- Prepublication history for this paper is available online. To view these files, please visit the journal online (http://dx.doi. org/10.1136/bmjopen-2020046027).

Received 25 0ctober 2020 Accepted 21 July 2021

Check for updates

(c) Author(s) (or their employer(s)) 2021. Re-use permitted under CC BY-NC. No commercial re-use. See rights and permissions. Published by BMJ.

Department of Social and Clinical Pharmacy, Charles University Faculty of Pharmacy in Hradec Kralove, Hradec Kralove, Czech Republic

Correspondence to

Dr Josef Maly;

malyj@faf.cuni.cz

\section{ABSTRACT}

Introduction Surgical site infection (SSI) is a potential complication of surgical procedure. SSI after implant surgery is a disaster both for patients and surgeons. Although predictive tools for SSI are available, none of them estimate early infection based on inflammatory blood parameters. The inflammatory process can be measured using several parameters including interleukin-6, C reactive protein, neutrophil to lymphocyte ratio, white cell count, erythrocyte sedimentation rate or procalcitonin. This systematic review aims to determine whether inflammatory blood parameters could be used as significant predictive factors for SSI after primary hip or knee arthroplasty.

Methods and analysis A systematic review of randomised controlled trials, cross-sectional studies, case-control studies and cohort studies, published in English, will be searched in the following electronic bibliographic databases: MEDLINE, Embase, PubMed, Cochrane Central Register of Controlled Trials and Web of Science. Studies performed in adult patients of all ages who underwent knee or hip arthroplasty, studies containing data on the risk/prognostic factors for preknee or postknee or hip arthroplasty SSI and studies with a minimum follow-up of 30 days after surgery will be included. A standardised form will be used to extract data from the included studies comprising study characteristics, participant characteristics, details of the intervention, study methodology and outcomes. Quality Assessment of Diagnostic Accuracy tool, second version, and Standards for Reporting of Diagnostic Accuracy Studies checklist will be used to assess risk of bias. Heterogeneity will be assessed using Cochran $\chi^{2}$ statistic and $I^{2}$ statistics where applicable. Grading of Recommendations Assessment, Development and Evaluation and Preferred Reporting Items for Systematic Reviews and Meta-Analyses guidance will be used to report findings.

Ethics and dissemination № ethics approval is required. The findings will be disseminated at national and international scientific sessions, also to be published in a peer-reviewed journal.

PROSPERO registration number CRD42020147925.
Strengths and limitations of this study

- The protocol reduces the feasibility of duplication, clarifies the methods and processes used, reduces potential review authors' biases and allows peer review of planned methods. The protocol offers the highest level of evidence for informed decisions based on this systematic review.

- To the best of our knowledge, this will be the first systematic review assessing the prognostic value of routine screening of inflammatory blood parameters for surgical site infection (SSI) in primary hip or knee arthroplasty.

- The scarcity of studies focused on patients after primary knee or hip arthroplasty containing data on the risk/prognostic factors for SSI with a minimum follow-up of 30 days, an expected heterogeneity in design, varying methodological quality across studies and limitation of review search to English language studies may hinder meta-analysis and interpretation of findings.

\section{INTRODUCTION}

Surgical site infection (SSI) is a potential complication following surgical procedure. ${ }^{1}$ The presence of SSI leads to an increase both in morbidity and in mortality rates, thus contributing to higher healthcare costs. ${ }^{23}$ In orthopaedics, SSI after implant surgery is a disaster both for the patients and surgeons. ${ }^{4}$ SSI is defined as an infection occurring up to 30 days after surgery (or up to 1 year after surgery in patients receiving implants) and affecting either the incision or deep tissue at the operation site. ${ }^{5}$ SSI prevalence may be reduced by various preoperative and postoperative measures. One of the measurements in which SSI is affected is antibiotic prophylaxis. ${ }^{67}$ It is desirable to adjust antibiotic prophylaxis length for arthroplasty, according to individual SSI risk estimated by 
appropriate processes. Although predictive tools for SSI, such as American College of Surgeons surgical risk calculator, or The National Healthcare Safety Network risk index are available, none of them estimate early infection based on inflammatory blood parameters (IBP).$^{89}$ In the acute phase, cells of the immune system migrate to the site of injury in a carefully orchestrated sequence of events mediated by cytokines and acute phase proteins. ${ }^{10}$ The inflammatory process can be measured applying one of the several biochemical markers, including interleukin-6 (IL-6), C reactive protein (CRP), neutrophil to lymphocyte ratio (NLR), white cell count (WCC), erythrocyte sedimentation rate (ESR) or procalcitonin (PCT). NLR is an easy, cheap, widely used, robust and convenient biomarker during inflammation, and has been shown to predict outcomes in cardiovascular diseases. ${ }^{11} 12$ After a surgical trauma neutrophil blood levels via cytokines are increased, and the lymphocyte count is decreased. ESR is the rate how quickly red blood cells settle at the bottom of test tube with blood sample. In case of disease, high ESR is commonly due to an increased protein level in the blood, such as the increase of acute phase proteins in inflammatory diseases. The ESR is nonspecific and, therefore, of little diagnostic value, but serial tests enable to follow the progress of the disease more efficiently. CRP, also known as nonspecific acute phase response marker, is instigated by tissue damage, infection, inflammation and malignancy. CRP is under control by IL-6 originating at the site of pathology. Serum concentration starts to rise within 6 hours, peaking in around 48 hours, from the time the given pathology occurs. CRP is still the most widely used biomarker for diagnosing postoperative infections. ${ }^{12}{ }^{13}$ PCT is one of the several bloodstream biomarkers investigated as an early predictor of sepsis. It is produced rapidly in response to bacterial stimuli but not in systematic inflammatory response syndrome of non-infectious origin. ${ }^{12}$ IL-6 is one of the polypeptides secreted by cells triggered in response to injury, in the broad category of small proteins cytokines. The role of cytokines is to organise a concerted response to tissue trauma, involving cells recruited to the injury site. IL-6 is classified as the inflammation cytokine of acute phase. To better understand the clinically significant responses to surgical trauma, it is important to elucidate the underlying mechanisms that regulate the inflammatory response to this type of tissue injury. The native immune system recognises bacteria through pattern recognition receptors, such as toll-like receptors. These receptors stimulate, among others, the secretion of IL- 6 , which is also stimulated by IL- $1(\alpha / \beta)$ and the tumour necrosis factor $(\alpha / \beta){ }^{11}$ The present systematic review will primarily assess the response of IBP to trauma caused by orthopaedic surgery, and evaluate the clinical utility of quantitative measurements of IBP as predictive factors for infection. To our knowledge, there is no published systematic review addressing the same question. Following the Preferred Reporting Items for Systematic Reviews and Meta-Analyses (PRISMA)
Protocol checklist ${ }^{14}$ as guidance, we propose a systematic and reproducible strategy to query the available literature.

\section{METHODS AND ANALYSIS}

The search strategy will be conducted according to the available resources, promoting methodological transparency and improving the reproducibility of the results and evidence synthesis. Additionally, using the Population, Intervention, Comparison, Outcome and Study design (PICOS) strategy, we elaborated the guiding question of this review in order to ensure the systematic search of available literature: Can IBP (IL-6, CRP, NLR, ESR, WCC, PCT) be effective as predictive factors for SSI in knee or hip arthroplasty? The planned period to compose this review study is from 1 September 2019 to 30 September 2021.

\section{Inclusion criteria for study selection}

A summary of the participants, interventions, comparators and outcomes considered, as well as the type of studies was made according to PICOS strategy.

\section{Types of studies}

Cross-sectional, cohort, case-control, randomised controlled trial study designs will be included, limited to publications in English language. Case reports will be excluded.

\section{Types of participants}

Studies with adult patients of all ages who underwent primary hip or knee arthroplasty will be included. The minimum follow-up after the operation must be 30 days. Patients with clinical signs of infection, neoplasia, inflammatory diseases (rheumatoid arthritis, Crohn's disease, HIV, etc) will be excluded.

Types of interventions or exposures

IBP determination such as IL-6, CRP, WCC, ESR, NLR and PCT.

\section{Types of compactors}

We will compare the prognostic value of IBP to each other.

\section{Types of outcomes measures}

The outcome measures will be the IBP that can be used as significant predictive factors for SSI development. SSI is defined as an infection occurring up to 30 days after surgery (or up to 1 year after surgery in patients receiving implants) and affecting either the incision or deep tissue at the operation site. ${ }^{5}$ Periprosthetic joint infection as subcategory to SSI will be defined according to the original diagnostic criteria proposed by the Musculoskeletal Infection Society Workgroup in 2011, ${ }^{15}$ the Infection Disease Society of America in 2013, ${ }^{16}$ the International Consensus Meeting in 2013, ${ }^{17}$ and finally according to the new proposed definition from 2018 using an evidencebased and weight-adjusted scoring system. ${ }^{18}$ 


\begin{tabular}{|c|c|}
\hline No & Search terms \\
\hline 1 & prosthesis joint infection or prosthesis related infection \\
\hline 2 & $\begin{array}{l}\text { prosthetic infection or inflammation or acute-phase reaction } \\
\text { or seroma or abscess or systematic inflammatory response } \\
\text { syndrome or sepsis }\end{array}$ \\
\hline 3 & wound infection \\
\hline 4 & surgical site infection or surgical wound infection \\
\hline 5 & $\begin{array}{l}\text { arthroplasty or arthroplasty, replacement or arthroplasty } \\
\text { replacement, hip or arthroplasty replacement, knee }\end{array}$ \\
\hline 6 & joint replacement \\
\hline 7 & $\begin{array}{l}\text { joint prosthesis or hip prosthesis or knee prosthesis or metal-on- } \\
\text { metal join prostheses }\end{array}$ \\
\hline 8 & joint arthroplasty \\
\hline 9 & risk factor or risk factors \\
\hline 10 & predictor \\
\hline 11 & inflammatory marker \\
\hline 12 & biological markers or hematologic tests \\
\hline 13 & leukocyte count \\
\hline 14 & blood sedimentation rate \\
\hline 15 & neutrophils \\
\hline 16 & lymphocytes \\
\hline 17 & neutrophil to lymphocyte ratio \\
\hline 18 & interleukin 6 \\
\hline 19 & $\mathrm{C}$ reactive protein \\
\hline 20 & procalcitonin \\
\hline 21 & 1 or 2 or 3 or 4 \\
\hline 22 & 5 or 6 or 7 or 8 \\
\hline 23 & 9 or 10 or 11 or 12 or 13 or 14 or 15 or 16 or 17 or 18 or 19 or 20 \\
\hline 24 & 21 and 22 and 23 \\
\hline 25 & limit to 24 (english language and yr="1902-Current") \\
\hline
\end{tabular}

For purpose of this table, search terms are presented without additional settings like 'focus, explode, multi-purpose', etc.

\section{Search methods for the identification of studies}

Electronic searches

We will conduct this systematic review and meta-analysis in accordance with the PRISMA guidelines. ${ }^{19}$ The following electronic bibliographic databases will be searched: PubMed (MEDLINE), Embase, Cochrane Central Register of Controlled Trials (Cochrane databases), Web of Science. The search terms will include both medical subject headings and keywords related to the topics about IBP or risk factors, or prosthesis-related infection or SSI and knee or hip arthroplasty. The sample of the search strategy for MEDLINE is presented in table 1. Similar search strategy will also be used to any other electronic database.

\section{Searching other resources}

Manual searches will be performed mainly to retrieve dissertations, ongoing trials, internal reports, etc, in order not to miss any relevant studies. Additionally, we will search the bibliographies of included publications manually for further, potentially eligible papers.

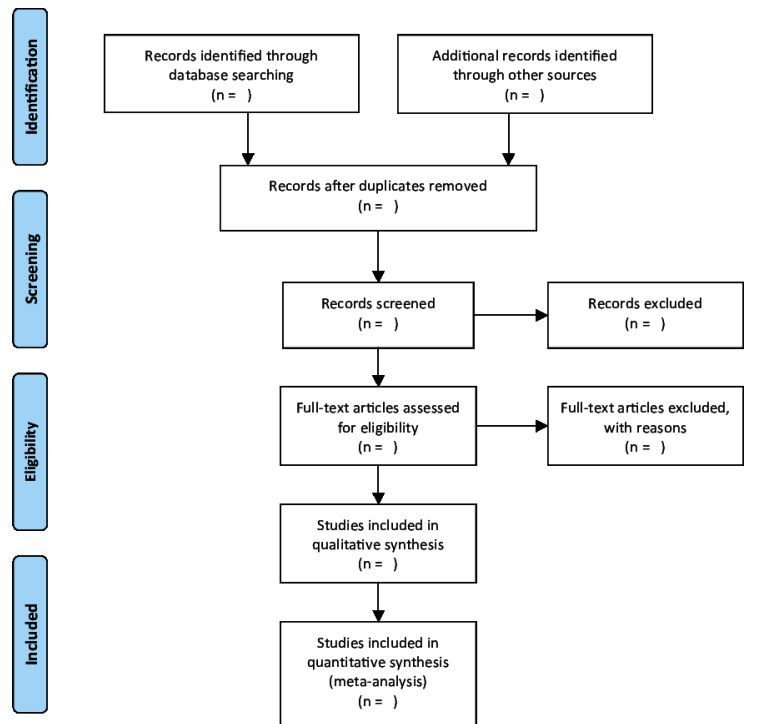

Figure 1 The PRISMA flow chart. ${ }^{19}$ PRISMA, Preferred Reporting Items for Systematic Reviews and Meta-Analyses.

\section{Data collection and analysis \\ Selection of studies}

All electronically and manually searched records will be merged to remove duplicate citations. Titles, abstracts and full-texts of studies retrieved using the search strategy will be screened independently through the EndNote software by at least two review authors (PD and ARP) to identify studies that potentially meet the inclusion criteria in the two-stage selection process. The initial screening of studies will be based on the information contained in their titles and abstracts, to be conducted by two independent investigators ( $\mathrm{PD}$ and $\mathrm{ARP}$ ). In case reviewers disagree, the article will be re-evaluated and if the disagreement persists, a third reviewer (KML) will be asked to make the final decision. Full-paper screening will be conducted by the same independent investigators. The reasons for literature excluding will be recorded. The details of the selection process are shown in the PRISMA flow chart (figure 1).

\section{Data extraction and management}

A standardised form will be used to extract data from the included studies for study quality assessment (Grading of Recommendations Assessment, Development and Evaluation (GRADE) algorithm $)^{20}$ and evidence synthesis. Extracted information will include study characteristics (design, settings, year of publication), participant characteristics (mean age, percentage of each gender), details of intervention (IBP with all characteristics), study methodology and outcomes (SSI, prosthesis-related infection, risk/prognostic factors, limitations). Missing data will be discussed with the authors of studies, where applicable. Two independent Microsoft Excel spreadsheets will be elaborated for two reviewers (PD and ARP) to summarise the data from the included studies. Then, the spreadsheets will be combined into one. Disagreements will be resolved by a third investigator (KML). 
Assessment of risk of bias in included studies

Quality Assessment of Diagnostic Accuracy-2 tool ${ }^{21}$ and Standards for Reporting of Diagnostic Accuracy Studies checklist $^{22}$ will be used to assess the risk of bias in the included studies by two reviewers (PD and ARP). The disagreement in the risk of bias assessment or the level of evidence (GRADE) will be resolved by consensus.

\section{Dealing with missing data}

If the required data are unclear or not reported in any of the articles, the reviewer will contact the author by email, or mail to collect the missing data as completely as possible.

\section{Assessment of heterogeneity}

Heterogeneity will be assessed using Cochran $\chi^{2}$ statistic and $\mathrm{I}^{2}$ statistics where applicable.

\section{Data synthesis and analysis}

We will conduct a narrative synthesis for the findings from the included studies, structured around the type of intervention, target population characteristics, type of outcome and intervention content. One reviewer (PD) will synthesise the data. Three other reviewers will check the results and discuss any discrepancies to reach consensus. If there is substantial clinical homogeneity of the participants and assessment methods, meta-analysis will be conducted to estimate the risk/protectability of each IBP in relation to knee or hip arthroplasty.

\section{Assessment of publication bias}

The funnel plot will be used to judge whether a publication bias exists if at least 10 articles are included. We will investigate the (a)symmetry in (inverted) funnel plots which show the association between effect size and the accuracy of the study.

\section{Subgroup analysis}

When the assessment suggests significant heterogeneity, we will perform a subgroup analysis based on study-level characteristics including diagnosis of the knee or hip osteoarthritis, type of surgery (based on how invasive method was used), duration of surgery, use of antibiotic prophylaxis (with other characteristics including dose and timing), thrombosis prevention, average duration of follow-up, exact time of performed blood test (preoperatively/perioperatively/postoperatively), laboratory analysis (eg, immunoturbidimetric technique, ethylenediaminetetraacetic acid as anticoagulation), American Society of Anesthesiologists physical status classification system, etc. Statistical analyses will be performed using SPSS v.27.0.1.

\section{Grading the quality of evidence}

We will use the GRADE software to assess the quality of the included studies: bias risk; heterogeneity; publication bias; and other factors will be considered. The quality of the evidence will be graded using 'very low', 'low', 'moderate', or 'high'.
Patient and public involvement

No patient will be involved.

\section{DISCUSSION}

The reproducible and transparent procedure for the systematic review of the literature is one of the strengths of our proposed protocol. We precisely described the types of studies, participants, interventions, comparators and outcomes to be included, as well as the data sources, search strategy, data extraction processes, data analysis and synthesis, and assessment of risk of bias. By publishing the research protocol, we reinforce the clarity of the strategy and minimise the risk of bias, namely selective outcome reporting. To the best of our knowledge, this is the first systematic review assessing the prognostic value of routine screening of IBP for SSI after primary hip or knee arthroplasty. Although a few systematic reviews for diagnosis of periprosthetic joint infection were published in the past, their conclusions cannot be used for primary arthroplasty prognosis. ${ }^{23-26}$ Therefore, this review is solely focused on primary arthroplasty only. The findings may be applicable during preoperative measurements to assess the individual risk for SSI to each patient, particularly if any discrepancies in antibiotic prophylaxis occur, because it needs to be individualised for each patient during mass operation, such as arthroplasty. This review will cover a large number of databases, including sources for a manual search. The use of articles in English language only is a limitation of the review. Poor-quality studies or reduced number of studies and heterogeneity in results may lead to difficulties in interpreting findings and to make a recommendation.

Contributors Review conceptualisation was designed by PD and ARP. Methodology of study design and literature search strategies were developed by PD, ARP and KML. Design of study quality risk assessment tools, data extraction, data synthesis and statistical tests were developed by PD, ARP and KML. Project supervision was carried out by JM. PD wrote the manuscript draft according to PRISMA-P. Draft revision was undertaken by all the authors.

Funding PD as a PhD student is supported by Charles University (Project SVV 260 551).

Competing interests None declared.

Patient and public involvement Patients and/or the public were not involved in the design, or conduct, or reporting, or dissemination plans of this research.

Patient consent for publication Not applicable.

Provenance and peer review Not commissioned; externally peer reviewed.

Open access This is an open access article distributed in accordance with the Creative Commons Attribution Non Commercial (CC BY-NC 4.0) license, which permits others to distribute, remix, adapt, build upon this work noncommercially, and license their derivative works on different terms, provided the original work is properly cited, appropriate credit is given, any changes made indicated, and the use is non-commercial. See: http://creativecommons.org/ licenses/by-nc/4.0/.

\section{ORCID iDs}

Petr Domecky http://orcid.org/0000-0002-0566-8080

Anna Rejman Patkova http://orcid.org/0000-0002-8072-4708

Katerina Mala-Ladova http://orcid.org/0000-0001-6554-7339

Josef Maly http://orcid.org/0000-0002-6538-1639 


\section{REFERENCES}

1 Namias N, Harvill S, Ball S, et al. Cost and morbidity associated with antibiotic prophylaxis in the ICU. J Am Coll Surg 1999;188:225-30.

2 Leaper DJ, van Goor H, Reilly J, et al. Surgical site infection - a European perspective of incidence and economic burden. Int Wound J 2004;1:247-73.

3 de Lissovoy G, Fraeman K, Hutchins V, et al. Surgical site infection: incidence and impact on hospital utilization and treatment costs. Am J Infect Control 2009;37:387-97.

4 Horan TC, Gaynes RP, Martone WJ, et al. CDC definitions of nosocomial surgical site infections, 1992: a modification of CDC definitions of surgical wound infections. Infect Control Hosp Epidemiol 1992;13:606-8.

5 Mangram AJ, Horan TC, Pearson ML, et al. Guideline for prevention of surgical site infection, 1999. centers for disease control and prevention (CDC) hospital infection control practices advisory committee. Am J Infect Control 1999;27:97-132.

6 Korol E, Johnston K, Waser N, et al. A systematic review of risk factors associated with surgical site infections among surgical patients. PLoS One 2013;8:e83743.

7 Bratzler DW, Dellinger EP, Olsen KM, et al. Clinical practice guidelines for antimicrobial prophylaxis in surgery. Am J Health Syst Pharm 2013;70:195-283.

8 Acs NSQIP surgical risk calculator. Available: https://riskcalculator. facs.org/RiskCalculator/index.jsp [Accessed 16 Sep 2019].

9 Culver DH, Horan TC, Gaynes RP, et al. Surgical wound infection rates by wound class, operative procedure, and patient risk index. National nosocomial infections surveillance system. Am J Med 1991;91:152S-7.

10 Germolec DR, Frawley RP, Evans E. Markers of inflammation. Methods Mol Biol 2010;598:53-73.

11 Bhat T, Teli S, Rijal J, et al. Neutrophil to lymphocyte ratio and cardiovascular diseases: a review. Expert Rev Cardiovasc Ther 2013;11:55-9.

12 Lowsby R, Gomes C, Jarman I, et al. Neutrophil to lymphocyte count ratio as an early indicator of blood stream infection in the emergency department. Emerg Med J 2015;32:531-4.

13 Daryapeyma A, Aarstad HJ, Wahlgren C-M, et al. Perioperative cytokine response to infection associated with elective arterial surgery. Vasc Endovascular Surg 2014;48:116-22.
14 Moher D, Shamseer L, Clarke M, et al. Preferred reporting items for systematic review and meta-analysis protocols (PRISMA-P) 2015 statement. Syst Rev 2015;4:1.

15 Parvizi J, Zmistowski B, Berbari EF, et al. New definition for periprosthetic joint infection: from the workgroup of the musculoskeletal infection Society. Clin Orthop Relat Res 2011;469:2992-4.

16 Osmon DR, Berbari EF, Berendt AR, et al. Diagnosis and management of prosthetic joint infection: clinical practice guidelines by the infectious diseases Society of America. Clin Infect Dis 2013;56:e1-25.

17 Parvizi J, Gehrke T, Chen AF. Proceedings of the International consensus on periprosthetic joint infection. Bone Joint J 2013;95B:1450-2.

18 Parvizi J, Tan TL, Goswami K, et al. The 2018 definition of periprosthetic hip and knee infection: an evidence-based and validated criteria. J Arthroplasty 2018;33:1309-14.

19 Moher D, Liberati A, Tetzlaff J, et al. Preferred reporting items for systematic reviews and meta-analyses: the PRISMA statement. PLoS Med 2009;6:e1000097.

20 Guyatt G, Oxman AD, Akl EA, et al. Grade guidelines: 1. introductiongrade evidence profiles and summary of findings tables. J Clin Epidemiol 2011;64:383-94.

21 Whiting PF, Rutjes AWS, Westwood ME, et al. QUADAS-2: a revised tool for the quality assessment of diagnostic accuracy studies. Ann Intern Med 2011;155:529-36.

22 Bossuyt PM, Reitsma JB, Bruns DE, et al. Stard 2015: an updated list of essential items for reporting diagnostic accuracy studies. BMJ 2015;351:h5527.

23 Lee YS, Koo K-H, Kim HJ, et al. Synovial fluid biomarkers for the diagnosis of periprosthetic joint infection: a systematic review and meta-analysis. J Bone Joint Surg Am 2017;99:2077-84.

24 Berbari E, Mabry T, Tsaras G, et al. Inflammatory blood laboratory levels as markers of prosthetic joint infection: a systematic review and meta-analysis. J Bone Joint Surg Am 2010;92:2102-9.

25 Li B, Chen F, Liu Y, et al. Synovial fluid $\alpha$-defensin as a biomarker for peri-prosthetic joint infection: a systematic review and meta-analysis. Surg Infect 2017;18:702-10.

26 Yoon J-R, Yang S-H, Shin Y-S. Diagnostic accuracy of interleukin-6 and procalcitonin in patients with periprosthetic joint infection: a systematic review and meta-analysis. Int Orthop 2018;42:1213-26. 